\title{
Análise da Relação Causal entre Imagem, Qualidade, Satisfação e Fidelidade: um estudo sobre a percepção do turista nacional no destino turístico Natal/RN
}

\author{
Casual Relationship Between Destination Image, Quality, Satisfaction and Loyalty: a study \\ on the brazilian tourists perception of Natal/RN touristic destination
}

\author{
Márcio Marreiro das Chagas ${ }^{1}$ \\ Sergio Marques $\mathrm{Jr}^{2}$
}

\section{Resumo}

O objetivo principal do estudo é analisar a relação causal existente entre a qualidade, a satisfação, a fidelidade e a imagem de destinos. Para tanto, se realizou um estudo exploratório-descritivo, do tipo Survey, com enfoque analítico quantitativo. A coleta de dados foi realizada através de questionário aplicado junto aos turistas nacionais no Aeroporto Internacional Augusto Severo e na Rodoviária da Cidade do Natal, principais locais de entrada e saída de turistas no local. A composição amostral foi aleatória simples chegando-se ao número final de 400 turistas entrevistados. Dentre os principais resultados, observou-se que são nove as dimensões da percepção de qualidade de destinos de Sol e Praia, ou seja, Praias e Facilidades, Equipamentos Públicos, Alimentos e Bebidas, Serviços de Transportes, Equipamentos do Hotel, Serviços do Hotel, Entretenimento e Atrativos, Acesso ao Hotel e Hospitalidade. Concluiu-se que a imagem exerce influência forte e direta tanto no processo de satisfação do consumidor quanto no de fidelização. As dimensões da qualidade encontradas também se apresentaram como fortes influenciadoras de ambos os fatores, sendo que a satisfação influencia de forma direta a fidelização ao destino.

Palavras-chave: imagem de destinos turísticos; comportamento pós-compra; qualidade.

\footnotetext{
Abstract

This study aims to analyze the causal relationship between quality, satisfaction, fidelity and destination image. Therefore, if conducted an exploratory, descriptive research with quantitative analytical approach. Data collection was performed by a questionnaire addressed to Brazilian tourists at the Augusto Severo International Airport and Bus station of

${ }^{1}$ Doutorando em Administração pela Universidade Federal do Rio Grande do Norte (UFRN). Bacharel e Mestre em Turismo (UFRN). Professor do Serviço Nacional de Aprendizagem Comercial (SENAC-RN) e Tutor do DETED do Instituto Federal de Educação, Ciências e Tecnologia do Rio Grande do Norte (IFRN). Email: marcio_marreiro@yahoo.com.br.

${ }^{2}$ Graduado em Engenharia Agronômica pela Universidade de São Paulo (USP). Mestre em Agronomia pela USP. Doutor em Agronomia pela Universidade Estadual Paulista Júlio de Mesquita Filho. Professor adjunto da Universidade Federal do Rio Grande do Norte (UFRN). Email: sergio@ct.ufrn.br.
} 
Natal, the main points of entry and exit of tourists. The composition was simple random sampling by reaching the final number of 400 tourists interviewed. Among the main results, it was noted that nine are the dimensions of perceived quality of "Sun and Sand" segment, that is, Beaches and Facilities, Public Equipment, Catering Services, Transportation, Hotel Equipment, Hotel Services, Entertainment \& Attractions, Access to the Hotel and Hospitality. Moreover, it was concluded that the tourism destination image exerts strong and direct influence on both factors, in the customer satisfaction process and loyalty, the dimensions of quality also claimed to be strongly influences both factors, and finally, the satisfaction directly influences the loyalty to the destination.

Keywords: destination image; behavioral intentions; quality.

\section{Introdução}

A atividade turística assume a cada dia sua participação hegemônica como um dos principais setores econômicos em escala global (UNEP, 2007; WTTC, 2007; MTUR, 2007). Esta realidade é encontrada em diversos locais que, em razão do propagado potencial econômico do segmento, almejam se lançar no desenvolvimento desta atividade. Dessa maneira, é perceptível o aumento geométrico do número regiões que se lançam na tentativa de se tornar em destinos turísticos e assim conseguirem os diversos benefícios gerados pelo setor. Diante deste contexto, detecta-se a presença da crescente competitividade no setor de turismo (RIMMIGTON; KOZAK 2000; VALLS, 1996; RITCHIE; CROUNCH, 2003).

Observa-se nesse cenário que diversos destinos estão se estruturando cada vez mais em bases gerenciais competitivas, aumentando a sua capacidade de atração e almejando, doravante, entrar no hall dos principais destinos receptores, o que implica na necessidade de informações e ações que promovam a diferenciação e aumento de competitividade do destino (RITCHIE; CROUNCH, 2003; CHEN; TSAI, 2006; YÜKSEL, 2001).

Nesta perspectiva competitiva, potencializa-se a importância do conhecimento de fatores como a qualidade, satisfação e fidelidade (ANDRIOTIS; AGIOMIRGIANAKIS; MIHIOTIS, 2007; ZABKAR; BRENCIC; DMITROVIC, 2009; HUI; WAN; HO, 2007; MENG; TEPANON; UYSAL, 2008) e principalmente a imagem de destinos (CHI; QU, 2008; BOSQUE; MARTÍN, 2008) que aparece como uma importante ferramenta estratégica no processo de diferenciação, aumento da competitividade, satisfação e fidelização do consumidor de turismo ao destino, culminando, por fim, em um satisfatório desenvolvimento 
turístico (CHI; QU, 2007; ECHTNER; RITCHIE, 1991; PIKE, 2002; GALLARZA; GIL; CALDERÓN, 2002; BOSQUE; MARTÍN, 2008).

É patente o crescimento da importância da imagem uma vez que esta é reconhecida como sendo um dos principais fatores influenciadores da escolha de um destino (CHI; QU, 2007; ECHTNER; RITCHIE， 1991; PIKE，2002; GALLARZA; GIL; CALDERÓN，2002; BOSQUE; MARTÍN, 2008. CHEN; TSAI, 2006), assim como pela satisfação e a possível repetição dele (CHI; QU, 2007; VALLS, 1996; PIKE, 2002).

Entretanto, não obstante a importância da qualidade, satisfação, fidelidade e imagem de destinos turísticos como fatores direcionadores de competitividade, estes não têm tido uma devida atenção na literatura. Embora, em outras áreas de estudo, o constructo satisfação, assim como os demais, já tenha sido amplamente trabalhada, nos estudos em turismo este item ainda possui um escasso material de referência, principalmente no que diz respeito ao desenvolvimento de modelos, métodos e instrumentos específicos da área, como afirmam Bedia, Fernández e López, (2007), Yilmaz (2009), Juwaheer (2004) Akbaba (2006) AlbaceteSáez, Fuentes-Fuentes e Lloréns-Montes (2007).

Essa situação pode ser melhor percebida quando se trata do exame da relação causal entre os constructos (CHI; QU, 2007; BOSQUE; MARTÍN, 2008; CHEN; TSAI, 2006; ZABKAR; BRENCIC; DMITROVIC, 2009; YOON; UYSAL, 2005; CORREIA; PIMPÃO, 2005). Nesse sentido, buscou-se neste estudo analisar qual a relação causal entre a imagem, a qualidade, a satisfação e a fidelidade, tendo como escopo o destino turístico Natal, capital do Estado do Rio Grande do Norte, sob a percepção do turista nacional. O objetivo foi o de contribuir para a diminuição da carência teórica na área, como também apresentar informações que possam ser utilizadas como subsídios para tomadas de decisões estratégicas para o destino turístico.

\section{Modelos de Avaliação da Relação entre Imagem, Qualidade, Satisfação e Fidelidade}

\subsection{Modelo de Yoon e Uysal (2005)}

Um dos modelos que discute a relação entre satisfação com o destino turístico e o processo de fidelização a ele é o desenvolvido por Yoon e Uysal (2003) que, diferentemente dos outros que serão vistos a seguir, não traz a discussão dessa relação com a inclusão do constructo 
imagem de destinos, diretamente. De fato, este modelo apresenta implicações tanto teóricas quanto gerenciais com relação ao desenvolvimento de reflexões sobre a relação satisfação, fidelidade e fatores pull e push, fatores esses que, por sua vez, impactam o processo de formação da imagem de destino turístico como afirmam Baloglu e McCleary (1999).

Este modelo parte da relação entre os fatores push (psicológicos) e pull (culturais) cuja relevância é respaldada pela importância dos estudos relacionados aos aspectos que motivam a escolha do turista de um determinado destino, como afirmam Yoon e Uysal (2003) entre outros. O primeiro é constituído por aqueles fatores intrínsecos ao indivíduo, ou seja, aqueles fatores psicossociais que provocam o interesse no turista em realizar uma viagem, e estão, por sua vez, relacionados a aspectos emocionais ou internos, ou seja, a necessidade de escape, as relações familiares, o relaxamento, interação social, novidade, prestígio, aprendizagem, descanso, aventura/entretenimento, experiência cultural entre outros (GUTIÉRREZ, 2005). Já o segundo é constituído por aqueles fatores eminentemente externos ao indivíduo que estimulam a escolha por um destino dentre todos que estão disponíveis para a sua viagem, por exemplo, os atributos da destinação (YOON; UYSAL, 2003; CORREIA; PIMPÃO, 2005).

A partir deste modelo observou-se a relação direta e positiva entre satisfação do turista e fidelidade ao destino presente em grande parte da literatura, como pode ser visto em $\mathrm{Chi}$ e $\mathrm{Qu}$ (2008), Bosque e San Martin (2008), Juwaheer (2004) e Akbaba (2006) entre outros. Além disso, este modelo apresenta a satisfação do turista como sendo negativamente afetado pelos fatores pull e não apresentando relação significativa com os fatores push, tendo este último uma relação direta e significativa com a fidelidade ao destino por parte dos turistas.

Nesse sentido, a fim de obter êxito em suas propostas de marketing, de acordo com Yoon e Uysal (2003), o destino turístico deveria levar em consideração os fatores pull que, como visto, fazem menção a aspectos externos ao indivíduo como os atributos apresentados pelo próprio destino com o objetivo de adequá-los ao público. Outra importante implicação deste modelo é o fato da fidelidade ao destino ser diretamente vinculada aos fatores push o que implica na oportunidade dos destinos em investir maciçamente em aspectos mais relacionados aos lados emocionais e afetivos do turista, objetivando a fidelização do turista ao destino. Neste ponto, percebe-se a grande proximidade com o papel do constructo imagem, observado em outros modelos, uma vez que este inclui fatores como esses apresentados. 


\subsection{Modelo de Chen e Tsai (2006)}

Outro modelo que propõe uma abordagem integrada da relação entre qualidade, satisfação e fidelidade à destinos turísticos é o desenvolvido por Chen e Tsai (2006) que, além dos constructos mencionados, inclui também a imagem de destinos (percepção geral do turista com relação a uma destinação), a exemplo das estruturas propostas por Bosque e San Martin (2008) e Chi e Qu (2008), entre outros. Além disto, o modelo inclui o constructo valor (Custo/benefício) na relação.

Diante de 10 hipóteses primárias levantadas, o modelo conseguiu significância apenas em metade delas. Desse modo, de acordo com Chen e Tsai (2006), a imagem influencia a percepção da qualidade e a propensão à fidelidade, em consonância com os estudos de Gallarza, Gil e Calderón (2002), Chi e Qu (2008), Bosque e San Martin (2008), Echtner e Ritchie (1991), Pike (2002), Valls (1996). No entanto, não possui significância estatística quando se coloca em perspectiva a relação entre ela [imagem], a percepção de valor e a satisfação. Este fato encontrado é respaldado por Bosque e San Martin (2008), entre outros.

A partir do estudo apresentado, concluiu-se que a qualidade da viagem influencia diretamente a percepção de valor ao passo que não exerce influencia significativa direta (apenas indireta mediada pela dimensão valor) no processo de fidelização e na satisfação do cliente, contrariando assim, estudos desenvolvidos por Taylor e Cronin (1994), Chi e Qu (2008) e Bosque e San Martin (2008), que defendem que a qualidade é antecedente da satisfação e fidelidade. Salienta-se que para Taylor e Cronin (1994), a qualidade, diferentemente da satisfação, não possui influencia na fidelidade do consumidor.

Por fim, Chen e Tsai (2006) firmam que a percepção de valor afeta a satisfação do consumidor enquanto que não influencia na fidelização dele, sendo que a fidelização está ligada ao grau de satisfação, de acordo com os estudos de Chi e Qu (2008), Bosque e San Martin (2008) e Taylor e Cronin (1994). Portanto, pode-se afirmar que a imagem e a satisfação são os fatores que exercem maior influencia no comportamento do consumidor no que se refere à fidelidade.

\subsection{Modelo de Chi e Qu (2008)}


De acordo com Chi e Qu (2008), a fidelidade ao destino pode ser explicada a partir da relação entre os constructos satisfação com os atributos do destino (compras, eventos, acomodações, acessibilidade, atrações, ambiente geral e alimentação), satisfação global e a imagem deste destino. Os autores investigaram a diferenciação lançada por Oliver (1993) onde é relatado que, embora os constructos satisfação com os atributos e satisfação global sejam relacionados, não são propriamente similares, carecendo, desse modo, de diferenciação e análise em separado. Além disso, defendem a imagem como um importante influenciador tanto na satisfação com os atributos como na satisfação global, assim como influenciadora do processo de fidelização.

A partir do modelo proposto pelos autores, observou-se que a imagem explica $51 \%$ da variação da satisfação com os atributos do destino, ao passo que a união da imagem com a satisfação com os atributos do destino confere um poder explicativo de $25 \%$ da satisfação global. Quando se adiciona o poder preditivo da imagem, satisfação com os atributos e satisfação global, chega-se a explicar $44 \%$ da variação no que concerne a fidelização do consumidor de turismo ao destino.

No modelo proposto por Chi e Qu (2008) observa-se que das 07 hipóteses originais lançadas, 06 puderam ser confirmadas enquanto que apenas 01 não apresentou significância estatística. Portanto, pode-se afirmar que a imagem e a satisfação com os atributos influencia positivamente a satisfação global, a imagem influencia positivamente a satisfação com os atributos do destino, a satisfação global influencia positivamente o processo de fidelização e a satisfação global com o destino media completamente a relação entre imagem e fidelidade. Não se pode afirmar que a satisfação global media completamente a relação entre a satisfação com os atributos e a lealdade ao destino, apenas se pode assegurar parcialmente essa relação.

Basicamente, neste modelo percebe-se o papel estratégico da imagem no gerenciamento de um destino turístico uma vez que esta influencia diretamente a satisfação com os atributos dele e a satisfação global, dimensões que influenciam diretamente a fidelidade.

\subsection{Modelo de Bosque e San Martin (2008)}

Para Bosque e San Martin (2008), o processo que culmina na fidelidade ao destino apresenta mais constructos a serem levados em consideração do que os encontrados nos modelos anteriores. Estes autores, ainda que levem em consideração a relação entre os constructos 
satisfação, imagem e fidelidade, acrescentam algumas outras variáveis como as emoções positivas e negativas, as expectativas e a teoria da disconfirmação que diz que a satisfação do consumidor é construída a partir da comparação entre as expectativas e o desempenho do serviço, havendo, assim, a disconfirmação ou não das expectativas do consumidor com relação ao serviço, culminando na satisfação ou não do turista. Além disso, embora as emoções possam ser consideradas como direcionadores da própria imagem, no modelo proposto foram separadas, em razão de seu poder explicativo elevado, a exemplo do modelo de Johnson et al (2001). A inclusão dessas novas dimensões culmina em um modelo teórico baseado nos aspectos psicológicos, de comportamento, tanto cognitivos como afetivos.

Dentre os resultados de 11 hipóteses levantadas e testadas no modelo, pode-se afirmar que as expectativas dos turistas possuem efeito direto e dominante junto à satisfação dele. Destaca-se que as expectativas também exercem influência direta nas emoções positivas (relação encontrada após a análise, não contemplada nas hipóteses primárias) percebidas pelos turistas, mas não apresenta significância estatística junto ao processo de disconfirmação. Esta última (disconfirmação), por sua vez, exerce influência direta junto às emoções positivas e negativas proporcionadas pelo destino. Entretanto, observou-se que não afetam a satisfação. Conforme proposto, as emoções positivas e negativas influenciam diretamente a satisfação.

O modelo apresenta ainda, o constructo imagem influenciando concomitantemente a fidelidade e as expectativas com relação à viagem. Entretanto, não demonstra influência na satisfação, ainda que atue como mediador da relação entre expectativas e fidelidade. Por fim, observa-se que as emoções positivas exercem influência direta junto à fidelidade (relação não inserida nas hipóteses primárias) e que esta é fortemente influenciada pela satisfação. Destaca-se que modelo se alinha à tendência atual observada em literatura em reconhecer a relação cognitiva-afetiva no processo de satisfação e fidelização como afirmam Chi e $\mathrm{Qu}$ (2008), Echtner e Ritchie (1991), Pike (2002) e Gallarza, Gil e Calderón (2002).

Faz-se mister ressaltar que é comprovada a importância da imagem no processo de formação de expectativas e na fidelização, mas não na satisfação. Este dado é contraditório aos encontrados nos estudos de Chi e Qu (2008), Gutiérrez (2005), Pike (2002), Gallarza, Gil e Calderón (2002) e Echtner e Ritchie (1991), entre outros, que afirmam que a imagem também influencia a satisfação. Outro resultado que merece destaque é a maior adequabilidade do uso 
das expectativas na explicação da satisfação do que a teoria da disconfirmação. Por fim, é pertinente frisar a influência de aspectos cognitivos na formação das emoções e o impacto delas na satisfação.

\subsection{Modelo de Zabkar, Brencic e Dmitrovic (2009)}

O modelo proposto por Zabkar, Brencic e Dmitrovic (2009) objetivou explicar a relação entre atributos do destino, a qualidade percebida, a satisfação e a fidelidade. É interessante observar que os autores procuram distinguir claramente a qualidade percebida da satisfação que, embora sejam diferentes, são tratados, grosso modo na literatura, da mesma maneira. Em outras palavras, os autores afirmam que embora se reconheça a diferença entre os constructos citados nas pesquisas realizadas, eles são operacionalizados de forma semelhante. No estudo proposto pelos autores, foram utilizados constructos reflexivos e formativos conjuntamente. $\mathrm{O}$ primeiro constructo (reflexivo) trata as dimensões como sendo latentes sendo as variáveis alocadas de acordo com as dimensões já consideradas. O segundo constructo (formativo) indica que é o conjunto de variáveis que explicam as dimensões, ou seja, é a partir do estudo das relações das variáveis que se observam as dimensões, ao contrário do primeiro onde as dimensões já existem e as variáveis que as constituem é que são colocadas em seu fator.

O estudo culminou na confirmação de todas as hipóteses levantadas. Verificou-se que a qualidade dos serviços afeta a percepção de qualidade. Logo, todas as demais sub-hipóteses relacionadas foram confirmadas, ou seja, a acessibilidade afeta a percepção de qualidade do destino, assim como as facilidades, atrações, pacotes disponíveis e atividades. Os serviços auxiliares são preditores da qualidade percebida. Verificou-se também que a qualidade dos serviços afeta diretamente a satisfação, sendo que esta apresenta relação direta e significante com a fidelidade. Esta última variável recebe influência direta da percepção de qualidade do destino. A partir do modelo observou-se que $61 \%$ da variância no constructo satisfação é explicada pela qualidade percebida do destino, ao passo que $75 \%$ da variância do constructo fidelização é explicada pela satisfação juntamente com a qualidade percebida.

Baseando-se nos modelos apresentados, procurou-se investigar a relação entre Qualidade, a Satisfação, a Fidelidade e a Imagem de Destinos, tendo como escopo, o destino turístico Natal, Rio Grande do Norte, cuja metodologia de pesquisa é apresentada a seguir. 


\section{Metodologia da Pesquisa}

O estudo apresentou-se como sendo exploratório e descritivo com relação aos seus objetivos. O método analítico foi o quantitativo, sendo a pesquisa realizada do tipo Survey. A investigação foi desenvolvida na Cidade do Natal, capital do Estado do Rio Grande do Norte, com o público constituído por turistas nacionais. A amostra foi probabilística, sendo o método amostral aleatório simples. O cálculo amostral foi realizado a partir da fórmula de população infinita, considerada pela Organização Mundial do Turismo (OMT, 2005), como a mais adequada para este tipo de pesquisa de mercado. O modelo matemático é expresso da seguinte forma:

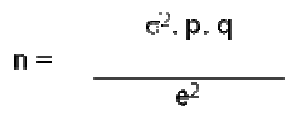

Fonte: OMT, 2005

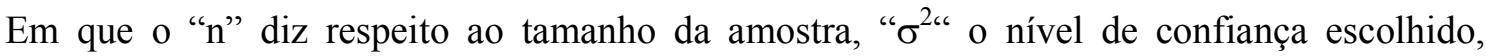
expresso em número de desvios-padrão, "p" a percentagem com a qual o fenômeno se verifica, "q" a percentagem complementar $(100-p)$ e "e $\mathrm{e}^{2 "}$ o erro máximo permitido. Para composição do cálculo foi considerado o nível de confiança de $95 \%$, a percentagem com a qual o fenômeno se verifica de $50 \%$, atribuição comum a trabalhos em Ciências Sociais Aplicadas conforme afirmam Hair Jr. et al (2006). O erro máximo permitido estimado foi de $5 \%$, como recomendado por Correia et al (2007). Dessa maneira, chegou-se ao número de 400 turistas definido como amostra final da investigação. O processo de coleta dos dados ocorreu entre os dias 01 e 30 do mês de Outubro de 2009.

O modelo de análise utilizado para o desenvolvimento do estudo foi baseado naquele proposto por Chi e Qu (2008), adaptado para destinos de Sol e Praia, como pode ser observado na Figura 01. 


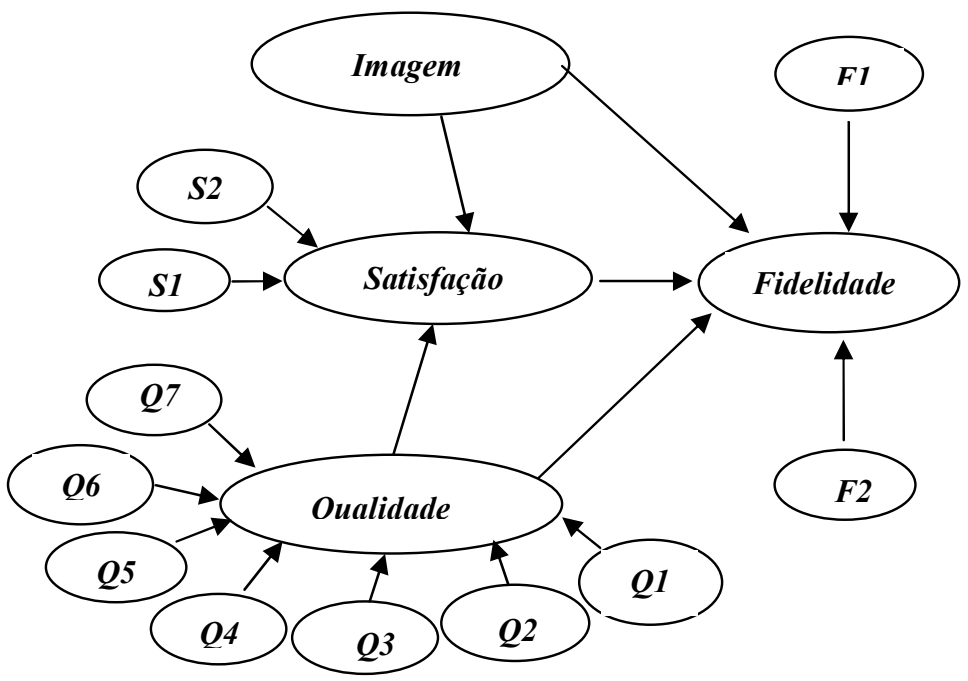

FIGURA 01 - Modelo adaptado para destinos de Sol e Praia baseado em Chi e Qu (2008) Fonte: Adaptado de Chi e Qu (2008)

Neste modelo, os direcionados da Qualidade do Destino são indicados pela letra "Q" acrescida de um número, onde Q1 se refere à Qualidade dos Meios de Hospedagens, Q2 a Qualidade dos Alimentos e Bebidas, Q3 a Qualidade das Praias, Q4 a Qualidade dos Equipamentos de Entretenimento, Q5 a Qualidade dos Atrativos Turísticos, Q6 a Qualidade da Infra-Estrutura Pública/Geral e Q7 a Qualidade dos Serviços de Transportes. Essas dimensões da qualidade foram selecionadas em razão de serem as dimensões comumente encontradas em diversos estudos de qualidade específicos do setor de turismo tais como Chi e Qu (2008), Hui, Wan e Ho, (2007), Chen e Tsai (2006), Rimmigton e Kozak (2000), Andriotis, Agiomirgianakis e Mihiotis, (2008), Yüksel (2001), Kozak (2000) entre outros.

Já os direcionadores da Satisfação do Turista com o Destino são representados por S1, que é Satisfação com Relação ao Custo-Benefício da Viagem, e S2 que representa a Satisfação Global com a Viagem, como encontrado em outras investigações realizadas por Yilmaz (2009), Hui, Wan e Ho, (2007), Juwaheer (2004) Akbaba (2006) Albacete-Sáez, FuentesFuentes e Lloréns-Montes (2007) e Chi e Qu (2008). Por fim, a Fidelidade possui como direcionadores o F1 que é a Intenção de Retornar ao Destino, e F2 que é a Propensão a Recomendá-lo à Outras Pessoas, como visto em nos estudos de Yilmaz (2009), Juwaheer 
(2004) Akbaba (2006) Albacete-Sáez, Fuentes-Fuentes e Lloréns-Montes (2007), Chi e Qu (2008), Hui, Wan e Ho, (2007), Bosque e San Martin (2008), Chen e Tsai (2006) entre outros.

O instrumento de pesquisa desenvolvido foi do tipo questionário, aplicado no Aeroporto Internacional Augusto Severo e na Rodoviária da cidade enquanto os turistas aguardavam o transporte de volta para a cidade de residência. O questionário foi baseado no instrumento SERVQUAL de Parasuraman, Zeithaml e Berry $(1985 ; 1988)$ e SERVPERF de Cronin e Taylor (1994), contendo 64 variáveis (incluindo perfil da amostra) adaptadas ao setor de turismo, conforme pode ser observado na Tabela 01. A escala utilizada foi do tipo Likert, de dez pontos, sendo o valor mínimo [01] e o máximo [10], tendo ainda a opção "Sem opinião" [99], para aqueles que não experimentaram o serviço ou não possuíam opinião formada. O questionário foi dividido entre as informações para a avaliação da qualidade, satisfação, imagem e fidelidade de forma que não fosse possível perceber qual dimensão estava sendo avaliada em cada momento, e o perfil do entrevistado. Os dados obtidos foram processados através do Statistical Package for the Social Sciences (SPSS) 17.0 para Windows. Para sua análise foram utilizadas estatísticas descritivas, em um primeiro momento, a fim de conhecer melhor os dados coletados, e logo depois de análises multivariadas, especificamente a Análise Fatorial Exploratória e a Análise de Regressão Linear Múltipla cujos parâmetros, métodos e testes estatísticos necessários para validação dos resultados são apresentados nas discussões a seguir.

\section{Resultados e Discussões}

\subsection{Validação da amostra}

A amostra da pesquisa foi constituída por turistas brasileiros de diferentes estados, tendo maior representatividade os estados das regiões Sudeste e Nordeste em consonância com os dados de demanda turística, elaborados pela Secretaria de Estado de Turismo do Rio Grande do Norte (SETUR, 2007). No que se refere à escolaridade, a maioria ficou dividida entre aqueles que afirmaram ter concluído o ensino médio $(22,6 \%)$, ter superior incompleto $(21,1 \%)$ e superior completo $(31,8)$, valores esses coerentes com a SECTUR/RN (2007). 
Quanto ao gênero, houve uma leve predominância do feminino $(56,2 \%)$ sobre o masculino $(43,8 \%)$ coerentemente com os dados do Instituto Brasileiro de Geografia e Estatística (IBGE, 2000) para a população brasileira, principalmente na faixa etária entrevistada que ficou essencialmente concentrada entre 26 e 50 anos (mais da metade da amostra), sendo que entre 26 a 35 anos encontrou-se a percentagem de $27,2 \%$ e entre 36 a 50 anos a percentagem de $25 \%$. Essa concentração na faixa de 26 a 50 anos pode ser explicada a partir do fator "estabilidade financeira". Geralmente, nessa faixa etária observa-se uma maior probabilidade de realização de viagens por parte dos participantes.

No que concerne a vinda a Natal, houve predominância daqueles que vieram com a família, seguida pelos que vieram com amigos, resultado semelhante aos das pesquisas de demanda turística realizadas pela SECTUR/RN (2007). Sobre a freqüência de visita a Natal, a maioria se concentrou naqueles que afirmaram nunca $(36,5 \%)$ ou quase nunca vir a Natal $(26,5 \%)$. A renda média familiar declarada por $35,3 \%$ dos entrevistados variou de $\mathrm{R} \$ 3.791,00$ a $\mathrm{R} \$ 7.582,00$, sendo seguida pelos que declararam ser $\mathrm{R} \$ 1.867,00$ a $\mathrm{R} \$ 3.790,00(28,2 \%)$.

Dessa maneira, pode-se concluir que a amostra pode ser considerada válida para fins deste estudo uma vez que as informações obtidas do perfil dos entrevistados são convalidadas por estudos como os desenvolvidos pela SECTUR/RN (2007) e IBGE (2000).

\subsection{Análise fatorial dos dados e validação de dimensões obtidas}

Um dos principais objetivos do trabalho consistiu em investigar quais as dimensões que compõem a qualidade de um destino turístico de "Sol e Praia", para depois utilizá-las no exame causal. Com vistas a investigar as dimensões, utilizou-se a Análise Fatorial, a fim de se investigar se as dimensões da percepção da qualidade do destino turístico, amplamente aceitas na literatura, se adéquam a realidade dos destinos de "Sol e Praia", especificamente Natal/RN, embora os resultados encontrados possam ser estendidos aos demais destinos de Nordestinos de sol e praia, em razão das características similares de desenvolvimento, como afirma Mazaro (2006). Nesta perspectiva, é necessário descobrir o percentual de contribuição de cada dimensão para a composição da percepção de qualidade e assim identificar prioridades.

Para o desenvolvimento da Análise Fatorial utilizou-se como método de extração dos fatores a Análise de Componentes Principais, juntamente com o método Varimax de rotação. Os 
fatores que obtiveram o Eigenvalue acima de 1,0 assim como as variáveis com cargas fatoriais acima de 0,4 foram considerados significantes e mantidos na análise.

Para a validação das dimensões obtidas através da Análise Fatorial foi aplicado o teste estatístico Alfa de Cronbach ( $\alpha$ ) a fim de avaliar a consistência interna de cada uma, e todas se mostraram adequadas. Conforme pode ser observada na Tabela 01, os valores obtidos de $\alpha$ foram bem acima do considerado aceitável pela literatura que é 0,7 , de acordo com Hair Jr. et al (2006), sendo o valor mínimo encontrado 0,834 e o máximo 0,968, ratificando a qualidade das variáveis escolhidas e dimensões encontradas. Nesse sentido, os resultados encontrados proporcionaram a validação do questionário utilizado e a confiabilidade das respostas contidas no banco de dados.

Com vistas a ratificar a significância dos resultados, como também a adequabilidade da amostra, foi aplicado o teste KMO (Kaiser-Meyer-Olkin) cujo valor atingiu 0,931, corroborado pelo Bartlett's Test of Sphericity de valor 18692,507, cuja significância foi zero. A carga fatorial e as dimensões da qualidade encontradas são apresentadas na Tabela 01:

TABELA 01 - Dimensões da qualidade de um destino turístico de "Sol e Praia"

\begin{tabular}{|c|c|c|c|c|}
\hline Dimensões e variáveis & Fatorial & Eigenvalue & Variância (\%) & $(\alpha)$ \\
\hline 1 Praias e facilidades & & 24,759 & 15,447 & 0,959 \\
\hline Limpeza da água e da areia & 0,620 & & & \\
\hline Segurança pública na praia & 0,685 & & & \\
\hline Equipamentos de apoio & 0,751 & & & \\
\hline Ambiente geral da praia & 0,746 & & & \\
\hline Outros serviços & 0,707 & & & \\
\hline Postos de vigilância e socorro & 0,825 & & & \\
\hline Equip. para prática de esportes & 0,743 & & & \\
\hline Placas indicadoras de risco, poluição e atividades náuticas & 0,785 & & & \\
\hline Adaptabilidade e serviços deficientes e crianças & 0,774 & & & \\
\hline Entretenimento diurno & 0,445 & & & \\
\hline Relação custo beneficio do restaurante & 0,497 & & & \\
\hline Relação custo beneficio dos Passeios & 0,560 & & & \\
\hline 2 Equipamentos públicos & & 4,188 & 13,737 & 0,968 \\
\hline Limpeza e higiene em vias públicas & 0,709 & & & \\
\hline Presença de mendigos, ambulantes, prostituição etc. & 0,736 & & & \\
\hline Sensação de segurança em vias públicas & 0,714 & & & \\
\hline Tranquilidade/Baixo nivel de Ruidos & 0,694 & & & \\
\hline Tráfego/Congestionamento & 0,665 & & & \\
\hline Acesso aos atrativos turísticos & 0,679 & & & \\
\hline Equip. públicos (lixeira etc) & 0,675 & & & \\
\hline Paisagem urbana & 0,723 & & & \\
\hline Qualidade paisagística natural & 0,784 & & & \\
\hline
\end{tabular}


3 Alimentos e Bebidas

Variedade dos restaurantes

Variedade dos pratos

Limpeza do restaurante

Ambiente Geral

Adaptabilidade para crianças e deficientes

Qualidade da comida

Profissionalismo dos funcionários dos restaurantes

Localização e acesso

\section{Serviços de transporte}

Conforto e equipamentos do aeroporto

Pontualidade dos vôos

Procedimentos de embarque e desembarque

Rede de transportes para saida do aeroporto

Postos de informações turísticas espalhados na cidade

Serviços financeiros, bancos

Táxis

5 Equipamentos do hotel

Segurança do hotel

Qualidade ambiental do hotel

Equipamentos do quarto

Estética do hotel

Adaptabilidade para crianças e deficientes

Qualidade dos serviços complementares do hotel

Relação custo beneficio

6 Serviços do hotel

Limpeza do hotel

Profissionalismo dos funcionários do hotel

Qualidade do restaurante do hotel

Avaliação dos passeios

7 Entretenimento e atrativos

Comercio local/Shopping

Relação custo beneficio do entretenimento local

Diversidade de atrativos e passeios

Guia de turismo e bugueiro

8 Acesso ao hotel

Localização e acesso ao hotel

9 Hospitalidade

Hospitalidade das pessoas

Total da Variância Explicada
2,915

0,849

0,855

0,805

0,725

0,479

0,767

0,723

0,664

12,832

0,951

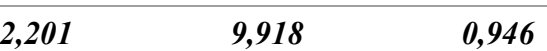

0,764

0,810

0,807

0,672

0,719

0,596

0,562

0,477

0,553

0,558

0,749

0,656

0,805

0,410

$\begin{array}{lll}1,796 & 7,206 & 0,914\end{array}$

0,651

$\begin{array}{lll}1,455 & 7,135 & 0,854\end{array}$

0,721

0,786

0,469

0,782
0,437
0,559
0,394

1,438

4,289

0,834

437

1,278

4,144

0,751

0,528

1,207

3,099

77,807

Nota: A avaliação dos atributos variou entre os valores (01) e (10), respectivamente pior e melhor desempenho Método de Extração: Análise de Componentes Principais

Método de Rotação: Varimax

KMO (Kaiser-Meyer-Olkin): 0,931

Bartlett's Test of Sphericity: 18692,507 (Sig, 0,000)

Fonte: Pesquisa de campo 
De acordo com a Tabela 01, observa-se que ao todo, nove (09) dimensões foram encontradas para explicar a percepção de qualidade em destinos de "Sol e Praia". Os valores encontrados são responsáveis por $77,807 \%$ da Variância Total Explicada, valor significativo e coerente com resultados encontrados em outras investigações. As nove (09) dimensões foram nomeadas em consonância com as variáveis que as compõem, sendo então denominadas de (1) Praias e Facilidades, (2) Equipamentos Públicos, (3) Restauração, (4) Serviços de Transportes, (5) Equipamentos do Hotel, (6) Serviços do Hotel, (7) Entretenimento e Atrativos, (8) Acesso ao Hotel e (9) Hospitalidade também em consonância com o modelo proposto pelo estudo e o encontrado na literatura como pode ser visto em Chi e Qu (2008), Hui, Wan e Ho, (2007), Chen e Tsai (2006), Rimmigton e Kozak (2000), Andriotis, Agiomirgianakis e Mihiotis, (2008), Yüksel (2001), Kozak (2000) entre outros.

Faz-se mister ressaltar que as dimensões Acesso ao Hotel e Hospitalidade não apresentam valores do teste Alpha de Cronbach em razão de conterem apenas uma variável cada, não sendo possível, assim, realização do teste.

Observou-se que o fator Praias e Facilidades (Variância 15,447) é o maior responsável pela percepção da qualidade em destinos de "Sol e Praia", resultado coerente com o esperado, uma vez que essa dimensão é considerada uma hipótese vinculada com essa percepção. Também foi observado que os Equipamentos Públicos assumiram a segunda dimensão mais impactante com variância explicada de 13,737\%, seguida por Alimentos e Bebidas (Variância 12,832\%), Serviços de Transporte (Variância 9,918\%), Equipamentos do Hotel (Variância 7,206\%) e Serviços do Hotel (Variância 7,135\%). As dimensões Entretenimentos e Atrativos (Variância 4,289\%), Acesso ao Hotel (Variância 4,144\%) e Hospitalidade (Variância 3,099\%) mostraram-se como os fatores menos influentes.

\subsection{Análise de regressão linear múltipla da satisfação}

Com vistas a analisar os fatores influenciadores da Satisfação Global do Consumidor Turístico (S3), foram agrupados em um único fator (utilizando-se a Análise Fatorial Exploratória) as variáveis Satisfação Global com a Passagem pelo Destino (S2) e a Satisfação Geral com a Relação Custo-Benefício (S1), criando então a variável S3. Esta (S3) foi a variável dependente que teve o comportamento examinado a partir do relacionamento existente com as demais variáveis explicativas. Já as variáveis independentes foram 
consideradas as nove (09) dimensões da qualidade obtidas a partir da análise fatorial, ou seja, Praias e Facilidades, Equipamentos Públicos, Alimentos e Bebidas, Serviços de Transportes, Equipamentos do Hotel, Serviços do Hotel, Entretenimento e Atrativos, Acesso ao Hotel e Hospitalidade em conjunto com a variável denominada Imagem do Destino, conforme modelo apresentado na Figura 01.

Para a realização da análise de Regressão Linear Múltipla, foi utilizado o método Stepwise, por ser considerado o mais adequado em razão do elevado número de variáveis analisadas e por evitar a multicolinearidade entre as dimensões do modelo. Destaca-se que também foi utilizado o método Forward, obtendo-se o mesmo resultado final, o que atesta a validade do método escolhido. O método Stepwise utilizou como parâmetro de inclusão de variáveis, os valores de probabilidade $\mathrm{p}$ iguais ou inferiores à 0,05 ao passo que para a exclusão, valores iguais ou superiores a 0,10 .

Da análise realizada encontrou-se um modelo com poder explicativo $\left(\mathrm{R}^{2}\right)$ de $86,5 \%$, com o teste F-ANOVA 311,988 e significância (Sig. 0,000), o que permite afirmar que as variáveis estatísticas testadas possuem influência sobre a variável dependente. O modelo então, como um todo, pode assim ser considerado como significativo. A ausência de multicolinearidade foi atestada pelos testes VIF e Tolerance, sendo todos os valores do primeiro inferiores a dez, limite para multicolinearidade inaceitável. Todos os valores do segundo teste obtiveram médias acima de 0,10, também utilizado como parâmetro máximo de multicolinearidade, como afirmam Hair Jr. et al (2006) e Corrar, Paulo e Dias Filho (2007).

Observa-se que o valor de 1,952 atingido pelo teste Durbin-Watson atesta a ausência de autorrelação assim como o teste $\mathrm{t}$ de Student com significância menor que 0,05 corroboram a afirmação da adequabilidade e significância estatística do modelo encontrado (CORRAR; PAULO; DIAS FILHO, 2007), que pode ser observado na Tabela 02. 
TABELA 02 - Fatores Influenciadores da Satisfação Global do Consumidor Turístico

\begin{tabular}{lccccc} 
Variáveis independentes & $\boldsymbol{\beta}$ não padronizado & Erro padrão & $\boldsymbol{\beta}$ Padronizado & Teste $\boldsymbol{S}$ & Sig. \\
\cline { 2 - 6 } (Constante) & $-4,438$ & 0,143 & -- & $-31,115$ & $<0,0001$ \\
Imagem & 0,503 & 0,016 & 0,751 & 31,381 & $<0,0001$ \\
Acesso ao hotel & 0,160 & 0,019 & 0,160 & 8,506 & $<0,0001$ \\
Praias e facilidades & 0,159 & 0,020 & 0,160 & 7,978 & $<0,0001$ \\
Equipamentos públicos & 0,123 & 0,020 & 0,124 & 6,035 & $<0,0001$ \\
Hospitalidade & 0,086 & 0,019 & 0,086 & 4,437 & $<0,0001$ \\
Entretenimento e atrativos & 0,078 & 0,019 & 0,078 & 4,130 & $<0,0001$ \\
Equipamentos do hotel & $-0,057$ & 0,019 & $-0,057$ & $-2,955$ & 0,0030 \\
Serviços de transportes & 0,051 & 0,019 & 0,051 & 2,663 & 0,0080
\end{tabular}

Nota: $\mathrm{R}^{2}=0,865 ; \mathrm{R}^{2}$ Ajustado = 0,862; $\mathrm{F}=311,988$ (Sig. 0,000); Durbin-Watson = 1,952.

Variáveis independentes $=$ Todas as listadas na tabela.

Variável dependente $=$ Satisfação Global do Consumidor Turístico.

Fonte: Pesquisa de campo.

Diante dos resultados apresentados na Tabela 02, conclui-se que a Imagem de Destinos ( $\beta$ 0,503) afeta forte e diretamente a Satisfação Global do Consumidor Turístico (S3), sendo a relação positiva. Isto leva a afirmação de que quanto melhor a imagem desenvolvida do destino, maiores são os níveis de satisfação com relação as atividades desfrutadas nele. Essa constatação corrobora com um extenso número de pesquisas científicas que culminaram na mesma conclusão tais como Chi e Qu (2007), Echtner e Ritchie (1991), Pike (2002), Gallarza, Gil e Calderón (2002), Valls (1996), entre vários outros.

Outra conclusão importante extraída é o fato da Imagem ( $\beta$ Padronizado 0,751$)$ ter influência aproximadamente oito vezes superior as quatro dimensões menos influentes, como foram a Hospitalidade ( $\beta$ Padronizado 0,086), Entretenimento e Atrativos ( $\beta$ Padronizado 0,078), Equipamentos do Hotel ( $\beta$ Padronizado -0,057) e Serviços de Transportes ( $\beta$ Padronizado 0,051), assim como aproximadamente cinco vezes superior as demais três dimensões que permaneceram no modelo, Acesso ao Hotel ( $\beta$ Padronizado 0,160), Praias e Facilidades ( $\beta$ Padronizado 0,160) e Equipamentos Públicos ( $\beta$ Padronizado 0,124). Portanto, conclui-se aqui, mais uma vez, o forte impacto exercido pela Imagem de Destino no processo de formação da Satisfação Global do Consumidor Turístico.

\subsection{Análise de regressão linear múltipla do processo de fidelização}


Para analisar a relação entre as dimensões da Qualidade, a Satisfação, a Imagem do Destino e a Fidelidade, utilizaram-se como variáveis independentes as mesmas dimensões originadas da percepção da qualidade do destino encontradas a partir da análise fatorial, juntamente com as variáveis Imagem do Destino e Satisfação Global do Consumidor Turístico. Como variável dependente se selecionou, através de análise fatorial, o fator Fidelidade (F3), constituído pelas variáveis Grau de Intenção de Recomendar o Destino a Amigos e Parentes (F2) e o Grau de Intenção de Retornar ao Destino (F1). Os parâmetros de análise utilizados para a Regressão Linear Múltipla foram os mesmos utilizados para a análise de regressão das variáveis direcionadoras de Satisfação. Os resultados do teste estatístico para esta análise podem ser observadas na Tabela 03 .

TABELA 03 - Fatores influenciadores da fidelização

\begin{tabular}{lccccc} 
Variáveis independentes & $\boldsymbol{\beta}$ não padronizado & Erro padrão & $\boldsymbol{\beta}$ Padronizado & Teste t & Sig. \\
\cline { 2 - 6 } (Constante) & $-3,387$ & 0,221 & --- & $-15,359<0,0001$ \\
Imagem & 0,384 & 0,025 & 0,573 & $15,395<0,0001$ \\
Satisfação com o destino & 0,319 & 0,035 & 0,319 & 9,040 & $<0,0001$ \\
Serviços do hotel & 0,093 & 0,017 & 0,094 & 5,607 & $<0,0001$ \\
Serviços de transportes & 0,050 & 0,016 & 0,050 & 3,212 & 0,0010 \\
Equipamentos públicos & 0,056 & 0,017 & 0,056 & 3,301 & 0,0010 \\
Entretenimento e atrativos & 0,038 & 0,015 & 0,039 & 2,490 & 0,0130 \\
Alimentos e Bebidas & 0,039 & 0,017 & 0,039 & 2,373 & 0,0180
\end{tabular}

Nota: $\mathrm{R}^{2}=0,910 ; \mathrm{R}^{2}$ Ajustado $=0,908 ; \mathrm{F}=566,282$ (Sig. 0,000); Durbin-Watson $=2,195$

Variáveis independentes $=$ Todas as listadas na tabela

Variável dependente $=$ Comportamento Pós-Compra Favorável ao Destino

Fonte: Pesquisa de campo

A partir dos dados apresentados na Tabela 03, percebe-se o impacto da Imagem junto ao processo de Fidelização ( $\beta$ Padronizado 0,573). A Imagem, desse modo, se mostra como o principal influenciador da Fidelização do Consumidor Turístico, embora seja perceptível que o binômio formado por ela e pela Satisfação Global, em termos absolutos, apresenta o maior impacto, o que é corroborado por diversos estudos como Chi e Qu (2007), Bosque e Martín (2008), Chen e Tsai (2006), Valls (1996) e Pike (2002). Os demais fatores apresentados demonstram poderes explicativos marginais quando comparados com as dimensões citadas, sendo eles, em ordem de influência, Serviços do Hotel ( $\beta$ 0,093), Equipamentos Públicos ( $\beta$ 
0,056), Serviços de Transportes ( $\beta 0,050)$, Alimentos e Bebidas $(\beta 0,039)$ e Entretenimento e Atrativos $(\beta$ 0,038). A figura 02 apresenta os principais resultados do estudo.

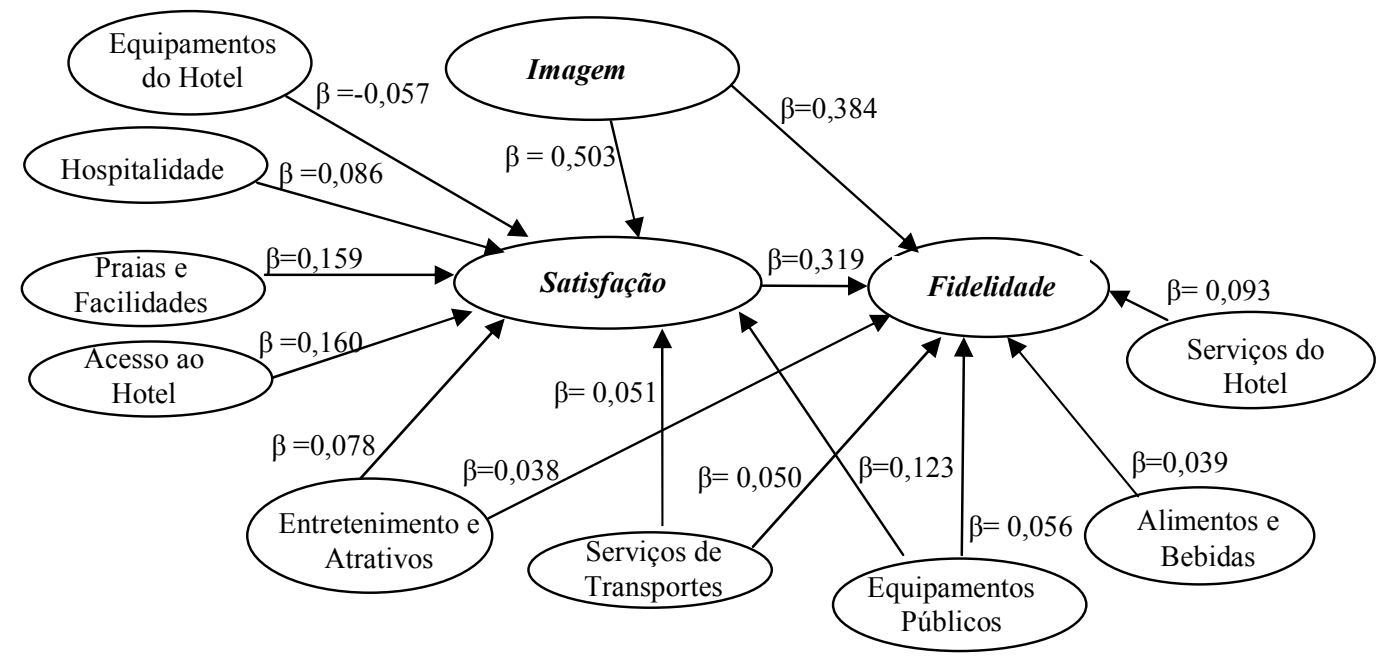

FIGURA 02 - Modelo encontrado pelo estudo Fonte: pesquisa de campo

\subsection{Implicações gerenciais}

A partir dos resultados encontrados pela investigação, diversas implicações gerenciais no que se refere ao planejamento e gestão de destinos turísticos de Sol e Praia, principalmente no que diz respeito ao aumento de competitividade, podem ser elucidadas. Dentre as mais importantes, conclui-se o forte impacto da Imagem de Destino na Satisfação, ratificando a importância dela [imagem] para o desenvolvimento de estratégias competitivas para o destino. Em outras palavras, faz-se importante ressaltar a necessidade de maior atenção para estratégias de formatação de uma imagem forte e atrativa para o destino, embasado em uma estratégia de marketing bem estruturada e embasada em fatores impactantes para o mercado consumidor

Além do impacto na Satisfação, a variável Imagem também se apresentou significativa para a Fidelização, assim como foi importante o próprio fator Satisfação para a fidelização. Nesse sentido, as variáveis Imagem e Satisfação parecem merecer significativa atenção a fim de 
prover o destino de maior competitividade no mercado. Dimensões como Acesso ao Hotel, Praias e Facilidades e Equipamentos públicos demonstraram também um importante papel no processo de satisfação do turista, implicando na necessidade de atuação centrada nesses aspectos primordiais para a satisfação, na percepção dos próprios consumidores. Destaca-se que essas dimensões impactam direta e fortemente a satisfação do turista e indiretamente na fidelização, intermediada pela dimensão satisfação, com exceção dos Equipamentos Públicos que também exercem influência direta na fidelidade ao destino. Com vistas à fidelização do público-alvo, cabe investir-se primordialmente na Imagem e na Satisfação, embora seja possível apontar dimensões relacionadas à hospedagem, atratividade e transporte como fatores importantes.

\section{Conclusões}

O estudo objetivou analisar a relação causal entre Qualidade, Satisfação, Comportamento PósCompra (Fidelidade) e a Imagem de Destinos. Nesse sentido, a primeira contribuição foi tentar uma aproximação de diversos conceitos que têm sido trabalhados, grosso modo, em separado, para assim se tentar chegar a informações que possam ser utilizadas no planejamento e gestão do destino turístico Natal, como também em outros de "Sol e Praia". Este tipo de estudo se faz relevante em razão do aporte de informações que pode prover aos stakeholders do setor, culminando, doravante, no aumento de competitividade do destino.

Outra contribuição foi ter analisado a relação causal entre diversos constructos para o desenvolvimento da teoria sobre comportamento do consumidor, o que colabora para o desenvolvimento de novas pesquisas que venham contemplar essa relação. Um dos principais aportes teóricos desse estudo tenha sido essa, uma vez que a partir do exame da relação causal existente entre tais constructos se faz possível ter uma idéia mais clara sobre o que exerce influência naqueles fatores no quais se quer agir.

Conclui-se, a partir dos resultados da pesquisa, que o instrumento utilizado para o desenvolvimento desta investigação se apresenta como válido e confiável para a medição da percepção da qualidade de destinos de "Sol e Praia" visto que todos os testes estatísticos realizados ratificam a validade e confiabilidade do questionário utilizado na pesquisa, assim como permite ainda, a exclusão de algumas variáveis sem perda significativa de poder 
preditivo das dimensões encontradas, embora a exclusão destas variáveis não venha a aumentar os índices de consistência interna ou confiabilidade.

Dentre os principais resultados, encontrou-se que a percepção da qualidade do destino turístico Natal/RN é composta por nove (09) dimensões principais que explicam aproximadamente $78 \%$ da variância total explicada do constructo. As dimensões encontradas que explicam a percepção da qualidade de destinos turísticos de "Sol e Praia", para a amostra investigada foram: Praias e Facilidades, Equipamentos Públicos, Restauração, Serviços de Transportes, Equipamentos do Hotel, Serviços do Hotel, Entretenimento e Atrativos, Acesso ao Hotel e Hospitalidade.

Do exame da relação entre os constructos lançados para a análise, ou seja, Imagem do Destino, Qualidade, Satisfação e Fidelidade obtiveram-se resultados respaldados pela literatura especializada. Dentre as principais constatações, a Imagem se apresentou como quesito primordial para a Satisfação Global do Consumidor em conformidade com as investigações de Chi e Qu (2007), Valls (1996), Pike (2002), Gallarza, Gil e Calderón (2002), Echtner e Ritchie (1991), demonstrando forte influência na explicação da variação na Fidelidade do Turista, em consonância com os estudos de Bosque e Martín (2008), Chi e Qu (2007), Valls (1996); Pike (2002); Gallarza; Gil; Calderón (2002), Echtner e Ritchie (1991). Nesse sentido, faz-se premente dar atenção especial ao item Imagem na composição da estratégia a ser desenvolvida para o destino.

\section{Agradecimentos}

Agradecemos a Maurício Nascimento Prazeres e Maria Auxiliadora Câmara de Araújo pela inestimável contribuição na pesquisa que originou este artigo.

\section{Referências}

AKBABA, Atilla. 2006. Measuring service quality in the hotel industry: A study in a business hotel in Turkey. Hospitality Management. Vol. 25, pp. 170-192.

ALBACETE-SÁEZ, Carlos A.; FUENTES-FUENTES, M. Mar; Lloréns-Montes; F. Javier. 2007. Service quality measurement in rural accommodation. Annals of Tourism Research, Vol. 34, No. 1, pp. 45-65. 
ANDRIOTIS, Konstantinos; AGIOMIRGIANAKIS, George; MIHIOTIS, Athanasios. 2008. Measuring tourist satisfaction: A factor-cluster segmentation approach. Journal of Vacation Marketing.Vol. 14 No. 3, p. 221-235.

BEDIA, Ana María Serrano; FERNÁNDEZ, María Concepción López; LÓPEZ, Raquel Gómez. 2007. Gestión de calidad y turismo: revisión e implicaciones para futuras investigaciones. Cuadernos de Turismo. no 20, p. 251-266.

BALOGLU, Seyhmus; MCCLEARY, Ken W. 1999. A model of destination image formation. Annals of Tourism Research. v. 26 n.4, p. 868-897.

BOSQUE, Ignacio Rodríguez Del; MARTÍN, Hector San. 2008. Tourist satisfaction: a CognitiveAffective Model. Annals of Tourism Research, Vol. 35, No. 2, pp. 551-573.

CHEN, Ching-Fu; TSAI, DungChun. 2007. How destination image and evaluative factors affect behavioral intentions? Tourism Management. V. 28, p. 1115-1122.

CHI, Christina Geng Qing; QU, Hailin. 2008. Examining the structural relationships of destination image, tourist satisfaction and destination loyalty: An integrated approach. Tourism Management. V. 29 , p. 624-638.

CORREIA, Antónia; PIMPÃO, Adriano. 2008. Decision-making processes of Portuguese tourist travelling to South America and Africa. International Journal of Culture, Tourism and Hospitality Research. V. 2, No. 4, p. 330-373.

CRONIN, J.; TAYLOR, S. 1994. Servperf versus Servqual: reconciling performance based and perceptions minus expectations measurement of service quality. Journal of Marketing, v. 58, n. 1, p.125-31.

CORRAR, Luiz J.; PAULO, Edilson; DIAS FILHO, José Maria. 2007. Análise multivariada: para cursos de administração, ciências contábeis e economia. São Paulo: Atlas.

ECHTNER, Charlotte M.; RITCHIE, J. R. Brent. 1991. The meaning and measurement of destination image. The Journal of Tourism Studies. Vol. 14, n. 1, p. 37-47.

GALLARZA, Martina G; GARCIA, Haydaer Calderon; SAURA, I. Gil. 2002. Destination image: towards a conceptual framework. Annals of Tourism Research, vol. 29, n. 1, p. 56-78.

GUTIÉRREZ, Héctor San Martín. 2005. Estudio de La imagen de destino turístico y el processo global de satisfacción: adopción de um enfoque integrador. Santander (ES). Tese Departamento de Administracion de Empresas de La Universidad de Cantabria.

HAIR, Joseph F. Jr. et al. 2006. Análise multivariada de dados. São Paulo: Bookman.

HUI, Tak Kee; WAN, David; HO, Alvin. 2007. Tourists' satisfaction, recommendation and revisiting Singapore. Tourism Management. Vol. 28, 965-975.

IBGE. s/d. População. Disponível em: <http;//www.ibge.gov.br>. Acesso em: 02 de Maio de 2010

JUWAHEER, Thanika Devi. 2004. Exploring international tourists' perceptions of hotel operations by using a modified SERVQUAL approach - a case study of Mauritius. Managing Service Quality. Vol. 14, n. 5, pp. 350-364.

JOHNSON, M. D. et al. 2001. The evolution and future of national customer satisfaction index models. Journal of Economic Psychology. v.22, p.217-245.

KOZAK, M. 2001. Comparative assessment of tourist satisfaction with destinations across two nationalities. Tourism Management. V. 22, p. 391-401. 
MENG, Fang; TEPANON, Yodmanee; UYSAL, Muzaffer. 2005. Measuring tourist satisfaction by attribute and motivation: The case of a nature-based resort. Journal of Vacation Marketing. V. 14, n. 1, p. 41-56.

MAZARO, Rosana Mara. 2006. Competitividad de destinos turísticos e sostenibilidad estratégica: proposición de un modelo de evaluación de factores y condiciones determinantes. Barcelona (ES). Tese do programa de doctorado en investigación e tecnicas de mercado (DITMUB) de la Universitat de Barcelona.

Oliver, R. L. Cognitive, affective, and attribute bases of the satisfaction response. 1993. Journal of Consumer Research. V.20, p.418-430.

OMT. 2005. Introdução a metodologia da pesquisa em turismo. São Paulo: Roca.

PIKE, Steve. 2002. Destination image: analysis a review of 142 papers from 1973 to 2000. Tourism Management. V. 23, n 05, p. 541-549.

PARASURAMAN, A.; V. ZEITHAML; L. BERRY. 1985. A conceptual model of service quality and its implication for future research. Journal of Marketing, v. 49, p. 41-50.

1988. Servqual: A multiple-item scale for measuring consumer perceptions of service quality. Journal of Retailing, v. 64, n. 1, p. 12-40.

RITCHIE, J.R. Brent; CROUCH, Geoffrey I. 2003. The competitive destination: a sustainability perspective. University of Calgary: Canadá.

RIMMINGTON, M.; KOZAK, M. 2000. Tourist Satisfaction with Mallorca, Spain, as an Off-Season Holiday Destination. Journal of Travel Research, Vol. 38, p. 260-269.

SECTUR/RN. s/d. Planejamento estratégico Sectur 2004-2007. Disponível em: $<$ http//:www.natal.rn.gov.br/>. Acesso em: 31 dez. 2006.

UNEP. s/d. Economic Impacts of Tourism. Disponível em: <http://www.uneptie.org/pc/tourism/susttourism/economic. $>$ Acesso em: 28 nov. 2007.

VALLS, Josep Francesc. 1996. Las claves del mercado turístico: cómo competir en el nuevo entorno. Bilbao: Deusto.

WTTC. s/d. Executive summary: travel \& tourism climbing to new heights: the 2006 travel \& tourism economic research. Disponível em <http://www.wttc.org/>. Acesso em 24 fev. 2007.

YILMAZ, Ibrahim. 2009. Measurement of Service Quality in the Hotel Industry. Anatolia: An International Journal of Tourism and Hospitality Research. Vol. 20, n, 2, pp. 375-386.

YÜKSEL, Atila. 2001. Managing customer satisfaction and retention: A case of tourist destinations, Turkey. Journal of Vacation Marketing. V. 7 No. 2, p. 153-168.

YOON, Y.; UYSAL, M. 2005. An examination of the effects of motivation and satisfaction on destination loyalty: a structural model. Tourism Management. V. 26, p. 45 - 56.

ZABKAR, Vesna; BRENCIC, Maja Makovec; DMITROVIC, Tanja. 2009. Modelling perceived quality, visitor satisfaction and behavioural intentions at the destination level. Tourism Management. p. $1-10$.

\section{Recebido em: 06/06/2010 (1 ${ }^{\mathrm{a}}$ versão) $25 / 10 / 2010$ ( $2^{\mathrm{a}}$ versão)}

Aprovado em: 03/11/2010 